\title{
An invisible touch: Body-related multisensory conflicts modulate visual consciousness
}

Roy Salomon ${ }^{1,2}$, Giulia Galli ${ }^{2,3}$, Marta Łukowska ${ }^{2,4}$, Nathan Faivre ${ }^{1,2}$, Javier Bello Ruiz ${ }^{1,2}$, Olaf Blanke $e^{1,2,5}$

${ }^{1}$ Center for Neuroprosthetics, School of Life Sciences, Ecole Polytechnique Fédérale de Lausanne, Geneva, Switzerland

${ }^{2}$ Laboratory of Cognitive Neuroscience, Brain Mind Institute, Ecole Polytechnique Fédérale de Lausanne, Geneva, Switzerland

${ }^{3}$ I.R.C.C.S. Fondazione Santa Lucia, Rome, Italy

${ }^{4}$ Consciousness Lab, Institute of Psychology Jagiellonian University, Cracow, Poland

${ }^{5}$ Department of Neurology, University Hospital Geneva, Geneva, Switzerland

Correspondence:

Roy Salomon,

Laboratory of Cognitive Neuroscience, Brain Mind Institute,

Ecole Polytechnique Fédérale de Lausanne,

SV BMI LNCO AAB201 (Batiment AAB)

Station15, CH-1015Lausanne, Switzerland

e-mail: roy.salomon@epfl.ch

Target journal: Neuropsychologia

Running title: Visuo-tactile conflicts affect visual consciousness 
Main text word counts: Abstract, 226 words; Main Text, 5799 words.

Figures 4.

Keywords: bodily self-consciousness, visual consciousness, multisensory integration, continuous flash suppression, body illusion, awareness, haptic robotics.

\begin{abstract}
The majority of scientific studies on consciousness have focused on vision, exploring the cognitive and neural mechanisms of conscious access to visual stimuli. In parallel, studies on bodily consciousness have revealed that bodily (i.e. tactile, proprioceptive, visceral, vestibular) signals are the basis for the sense of self. However, the role of bodily signals in the formation of visual consciousness is not well understood. Here we investigated how body-related visuotactile stimulation modulates conscious access to visual stimuli. We used a robotic platform to apply controlled tactile stimulation to the participants' backs while they viewed a dot moving either in synchrony or asynchrony with the touch on their back. Critically, the dot was rendered invisible through continuous flash suppression. Manipulating the visual context by presenting the dot moving on either a body form, or a non-bodily object we show that: (i) conflict induced by synchronous visuo-tactile stimulation in a body context is associated with a delayed conscious access compared to asynchronous visuo-tactile stimulation, (ii) this effect occurs only in the context of a visual body form, and (iii) is not due to detection or response biases. The results indicate that body-related visuo-tactile conflicts impact visual consciousness by facilitating access of non-conflicting visual information to awareness, and that these are sensitive to the visual context in which they are presented, highlighting the interplay between bodily signals and visual experience.
\end{abstract}




\section{Introduction}

Empirical investigations of consciousness have become a focus of study in cognitive neuroscience. Two important subfields of research have recently evolved: the study of bodily self-consciousness, investigating how the feeling of the self as a unified entity in the body arises from the integration of multisensory bodily signals (Blanke, 2012; Blanke, et al., 2014; Ehrsson, 2007) and perceptual consciousness, focusing on the neural correlates of perceptual experiences (Dehaene, Changeux, Naccache, Sackur, \& Sergent, 2006; Rees, 2007).

The large majority of investigations in perceptual consciousness to date have focused on the visual modality and benefited from a large number of psychophysical methods to render stimuli invisible (Dehaene \& Changeux, 2011; Dubois \& Faivre, 2014; Kim \& Blake, 2005). Paradigms making visual stimuli unconscious have been used to understand the modulators of conscious access, as well as the extent of unconscious processing (Kouider \& Dehaene, 2007; Mudrik, Faivre, \& Koch, 2014; van Gaal \& Lamme, 2012). However, following early suggestions of encapsulation and modularity of visual processing (Fodor, 1983; Zeki \& Bartels, 1998) there have been few attempts to study how bodily signals affect visual awareness (Faivre, Salomon, \& Blanke, 2015). Yet, the fact that we experience a multisensory world around us, and not distinct 
unimodal worlds serves as a premise for a multimodal character of sensory processing and perceptual consciousness (Bayne, 2002; Deroy, Chen, \& Spence, 2014; Driver \& Noesselt, 2008). While early theories of consciousness have proposed that multisensory integration cannot be achieved without awareness of the stimuli (Baars, 2002), several studies have now revealed multisensory integration in the absence of awareness. This has been shown in the olfactory (Arzi, et al., 2012; Zhou, Jiang, He, \& Chen, 2010), tactile (Lunghi \& Alais, 2013; Lunghi, Binda, \& Morrone, 2010; Lunghi, Morrone, \& Alais, 2014), auditory (Alsius \& Munhall, 2013; Conrad, Bartels, Kleiner, \& Noppeney, 2010; Faivre, Mudrik, Schwartz, \& Koch, 2014), vestibular (Salomon, Kaliuzhna, Herbelin, \& Blanke, 2015) and proprioceptive (Salomon, Lim, Herbelin, Hesselmann, \& Blanke, 2013) domains, suggesting that different multisensory signals below perceptual thresholds are integrated.

Such unconscious interactions between multimodal signals are of special interest as they are thought to underlie bodily self-consciousness (Blanke, 2012). Indeed, the experimental manipulation of bodily self-consciousness in healthy subjects typically involves multisensory conflicts notably by matching tactile stimulations on one's real body with synchronous visual stimulations seen on an avatar's body or rubber hand (Botvinick \& Cohen, 1998; Ehrsson, Spence, \& Passingham, 2004; Lenggenhager, Tadi, Metzinger, \& Blanke, 2007; Salomon, Lim, Pfeiffer, Gassert, \& Blanke, 2013). For example, in the fullbody illusion (FBI), participants feel a tactile stimulation on their body, while seeing it on a virtual body located 2 meters away, which results in a spatial conflict between the avatar and the participant's physical body. When the viewed and felt visuo-tactile stimulation are synchronous, the multisensory conflict is enhanced, as participants feel the touch on their backs but see it at another spatial location (I feel touch on my body and see synchronous touch on the avatar's body). Such changes do not occur when the visuo-tactile stimulation is asynchronous (I feel touch on my body and see unrelated touch on the avatar's body). Importantly, these altered states of bodily self-consciousness are further modulated by the presence of a bodily form, as changes in bodily self-consciousness are smaller or absent if the viewed touch is presented on an object (I feel touch on my body and see related or unrelated movement on an object) (Aspell, Lenggenhager, \& Blanke, 2009; Evans \& Blanke, 2012; Lenggenhager, et al., 2007; Tsakiris, Carpenter, James, \& Fotopoulou, 2010; Tsakiris 
\& Haggard, 2005). Here we refer to the conflict arising from synchronous visuo-tactile stroking presented on a body (related to the RHI and FBI) as a body-related multisensory conflict. The presence of a bodily form has also been shown to impact cross-modal congruency effects (Aspell, et al., 2013; Aspell, et al., 2009; Pavani, Spence, \& Driver, 2000; Salomon, van Elk, Aspell, \& Blanke, 2012), as well as tactile acuity in the visual enhancement of touch (Kennett, Taylor-Clarke, \& Haggard, 2001; Taylor-Clarke, Kennett, \& Haggard, 2002), suggesting a role for the visual body context in visuo-tactile interactions. Yet, while many studies have investigated the role of such bodily visuo-tactile conflicts on bodily selfconsciousness there has been no attempt to investigate how such conflicts affect visual consciousness.

Here we sought to determine whether body-related visuo-tactile conflicts and the presence of a body form would modulate access to visual consciousness. We used the continuous flash suppression (CFS) paradigm, in which highly salient stimuli presented to one eye prevent the visibility of a target stimulus presented to the other eye for extended periods of time through interocular rivalry (Tsuchiya \& Koch, 2005). After some time the target stimulus "breaks though" the interocular suppression and becomes visible. The time required to break suppression can be used as a dependent measure indicating unconscious processing (Gayet, Van der Stigchel, \& Paffen, 2014; Jiang, Costello, \& He, 2007; Mudrik, Breska, Lamy, \& Deouell, 2011; Salomon, Lim, Herbelin, et al., 2013; Stein \& Sterzer, 2014; Stein, Sterzer, \& Peelen, 2012). We employed virtual reality with a robotic haptic stimulation platform to stroke the participants' back (Duenas, et al., 2011; Pfeiffer, et al., 2013) while masking the visual stroking with continuous flash suppression. Participants were asked to indicate the color of a moving dot as soon as it broke suppression and thus became visible. Critically, as in the full body illusion, the dot could be moving synchronously or asynchronously with the tactile stroking on the back. In three experiments we investigated (i) if body-related visuo-tactile conflict affected the time required to break suppression (exp.1), (ii) if this effect was modulated by the presence of a body form vs. an object (exp.3), (iii) if the results could be due to detection or response biases (exp.2). Additionally, we replicated our results in a fourth experiment, using a withinsubject design, controlling more rigorously for low-level visual differences between the object and body stimuli, and assessing the specificity of multisensory stimulation vs. unimodal visual 
stimulation. In line with previous results on integration of bodily and visual signals in the absence of consciousness (Lunghi, et al., 2010; Salomon, Lim, Herbelin, et al., 2013; Salomon, Lim, Pfeiffer, et al., 2013) we predicted that CFS would be broken faster in the absence of bodyrelated visuo-tactile conflict (i.e. asynchronous stroking compared to synchronous stroking) but only in the presence of a visual body form.

\section{Methods}

\subsection{Participants}

Seventeen healthy subjects ( 8 males, mean age 22.9 years, $\mathrm{SD}=3.5$ years, range $18-29$ ) from the student population at Ecole Polytechnique Fédérale de Lausanne (EPFL) took part in the body CFS experiment and another 15 participants ( 9 men, mean age 28.5, $\mathrm{SD}=1.7$ years, range 25-31) participated in the control experiment. The third experiment included other 18 participants (13 males, mean age 22 years, $\mathrm{SD}=3.2$ years, range 18-29). The fourth experiment included 19 participants (10 males, mean age 22.1 years, $\mathrm{SD}=2.6$ years, range 18-26). All participants were right-handed, had normal or corrected-to normal sight and no psychiatric or neurological history. They were paid 20-25 CHF. All participants gave informed consent; the study was approved by the ethics committee of EPFL and was performed in accordance with the Declaration of Helsinki. Overall, one participant was removed from the analysis because of chance level performance in some conditions, one participant was removed due to haptic robot failure and three other participants were excluded as they could not complete the experiment (i.e. did not break suppression at all).

\subsection{Experiment 1: body CFS}

\subsubsection{Continuous flash suppression task}


Tactile stimulation was delivered by a custom-built haptic robot system (Duenas, et al., 2011; Salomon, Lim, Pfeiffer, et al., 2013). The tactile stimulation used several pre-set motion profiles with variable velocity ranging from 0 to $67 \mathrm{~cm} / \mathrm{sec}(M=4.69 \mathrm{~cm} / \mathrm{sec}, \mathrm{SD}=$ $1.41 \mathrm{~cm} / \mathrm{sec})$. These were coupled with visual motion profiles of a moving dot at the corresponding location scaled for the visual display (in the synchronous condition), or at a non-corresponding location (in the asynchronous condition).. In the body continuous flash suppression experiment (body CFS), visual stimuli consisted of high-contrast dynamic noise patches suppressors ("Mondrians", as described in (Hesselmann \& Malach, 2011; Salomon, Lim, Herbelin, et al., 2013) and a target stimulus. The background picture was a picture of a man wearing a white t-shirt seen from the back (size H: 20,5 ${ }^{\circ} \mathrm{W}: 11,3^{\circ}$ ) (Ionta, et al., 2011), on top of which we displayed either the Mondrians or the target stimulus - a small dot moving up and down along the left side of the man's back. The dot could be either green or blue with equal luminance (size: H: 0.7 , W: $0.7^{\circ}$, blue: R 134 G 133 B 255, green: R 110 G 173 B 118). Stimuli were presented using ExpyVR, an in-house multimedia stimuli presentation software developed with Python 2.6 and the Open Graphics Library v.2.2 (available at http://lnco.epfl.ch/). The stimuli were projected onto a head-mounted display (HMD; VR1280 Immersion Inc., SXGA, $1280 \times 1024$ pxl, $60^{\circ}$ diagonal field of view, refresh rate $60 \mathrm{~Hz}$ ). Mondrians were rapidly $(10 \mathrm{~Hz})$ flashed to the participants' dominant eye (visual angle $\mathrm{H}: 8.9^{\circ}$, $\mathrm{W}: 1^{\circ}$ ), and the dot was presented concurrently to the other eye. There were four types of trials depending on the synchrony between the tactile and visual stroking (synchronous/asynchronous), and the color of the dot (blue/green). Each type of trial was repeated 40 times in a randomized order. In total, the 160 trials were grouped in 4 blocks of 7 minutes each. Each trial began with the simultaneous presentation of the Mondrians and the dot to separate eyes (see Figure 1). The contrast of the dot was ramped up from zero to full contrast over 7 seconds, so that it became fully visible without CFS after about 1 second. Participants were asked to press a key on a numeric keypad to indicate their response (blue or green) as soon as they saw the dot. After a response was provided, the trial continued for 10 seconds to allow the robot to return to its baseline condition. 

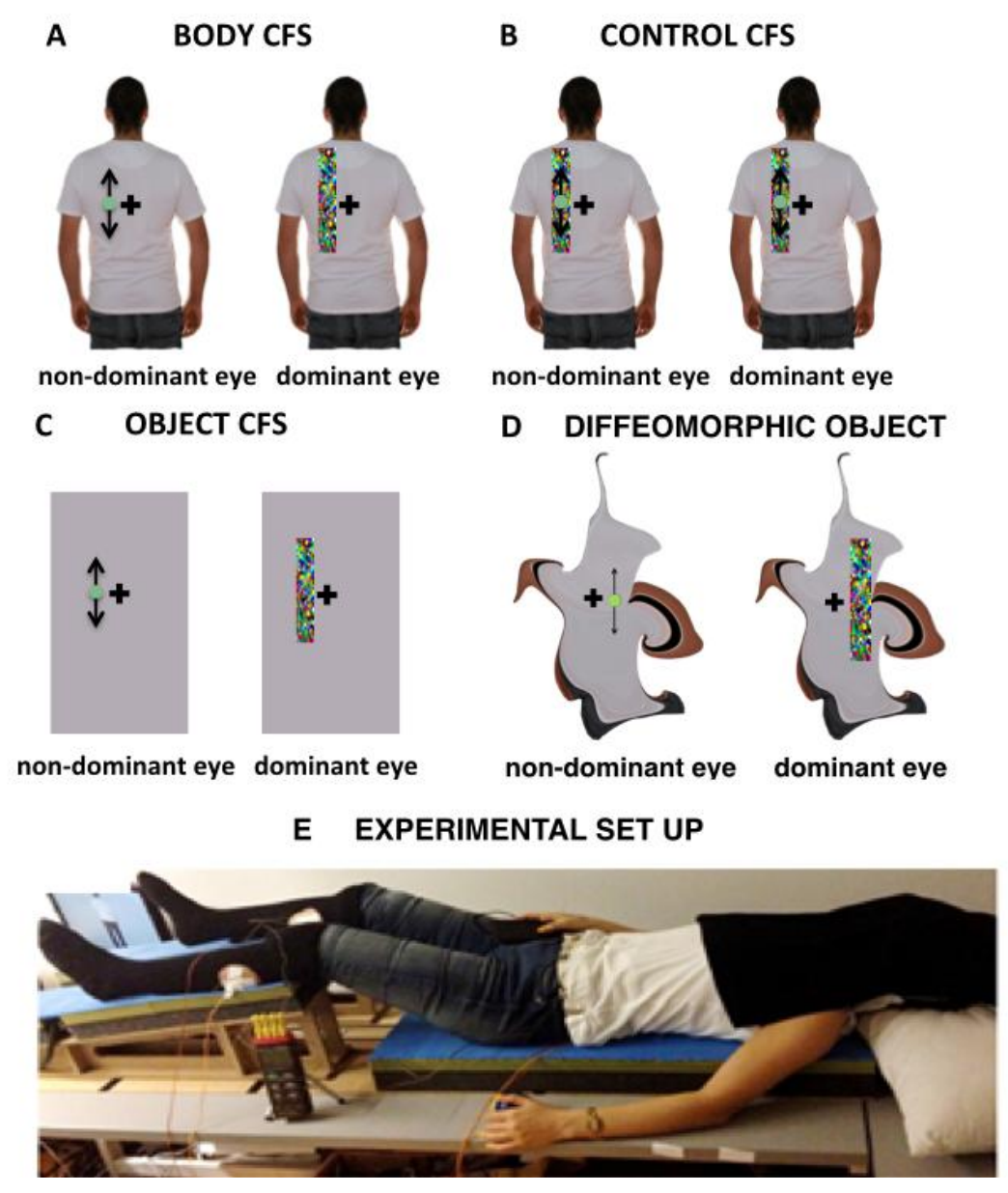

Figure 1. Visual stimuli in the three experiments (body CFS, control CFS, object CFS) and set up. A) body CFS: the body form and Mondrians were displayed to the dominant eye, while the moving dot was projected to the non-dominant eye (B) control CFS: the body form, Mondrians and moving dot were displayed to both eyes at the same time so that no interocular suppression occurred(C) object CFS: the object form and Mondrians were displayed to the dominant eye, while the moving dot was projected to the non-dominant eye. The black arrows indicate schematically the movement of the dot and were not present during the experiment. (D) Diffeomorphic object CFS: Object used in experiment $\mathbf{4}$ which is a diffeomorphic version of the body image thus well controlled for low level properties. (E) Schematic representation of the experimental set up. Participants were fitted with the HMD and asked to lie down supine on the stroking robot. 


\subsubsection{Procedure}

At the beginning of the experiment, participants were briefed about the stimuli they were going to experience and instructed about the task they were asked to perform. For a greater identification with the virtual body all participants were asked to wear a white t-shirt. Then, demographic data were collected and participants were tested for ocular dominance using the Miles test (Miles, 1930). Afterwards, they were fitted with the HMD and asked to lie down supine on the stroking robot. The experimenter placed their right hand on the response keypad, which was placed next to them. The participant's left hand was comfortably placed on the table matching the posture of the body image. Experimenter instructed participants to indicate the color of the moving dot by pressing one of two buttons and to respond as quickly as possible with the index or middle finger. During the experiment white noise was played through the headphones in order to mask noises of the robot. A short resting period was allowed between each block. No feedback was provided during the experiment. All subjects completed a short training session prior to the experimental blocks. Finally, we interviewed participants about their sensations during the experiment, i.e. difficulty of the task, awareness of the study purpose and differences in perceptual experience between synchronous and asynchronous conditions. The total duration of the experiment was about 1 hour.

\subsection{Experiment 2: control}

We designed a control experiment (control continuous flash suppression) to check for potential differences in detection time due to biases in response or detection criteria (e.g. shorter reaction

times, see (Salomon, Lim, Herbelin, et al., 2013; Stein, Hebart, \& Sterzer, 2011). The control experiment was identical to the main experiment except that the target images were blended into the Mondrians and presented to both eyes. Hence, in the control experiment, there was no interocular suppression: we projected both the Mondrian and the dot at the same time to both eyes together. Crucially, comparison of the results from the control CFS and body CFS experiments would allow one to test if the results in the body CFS experiment are exclusive to the visual suppression condition or may reflect a more general bias (Stein \& Sterzer, 2014; Stein, et al., 2012).

\subsection{Experiment 3: object CFS}


In Experiment 3 (object continuous flash suppression), participants performed a continuous flash suppression task as in Experiment 1. However, the background image on which the Mondrians and the dot were displayed was a body-sized object, instead of the virtual body. The object was a rectangle with the same color as the region on which the dot was presented in the body experiment (RGB: 187, 179, 179) and of the same size as the body template used in the previous experiments. All other parameters and procedures were identical to Experiment 1.

\subsection{Experiment 4: within-subject body-diffeomorphic object-visual only CFS}

In Experiment 4 (within-subject replication), participants performed a task as in the previous experiments, with one exception that the dot and Mondrians were presented on the right side of the avatar's body and stroking was delivered to the right side of participant's back. This experiment included three conditions: the body condition was identical to that of Experiment 1. In the object condition, to avoid possible confounds relating to the low level features of the object stimulus, we employed a diffeomorphic transformation on the body image (see Figure 1D) that preserves the basic perceptual properties of the image while removing its meaning (Stojanoski \& Cusack, 2014). Finally, a unimodal visual condition was introduced. A new HMD device was employed in this experiment, as the previous HMD was inoperative. We used an Oculus Rift DK, http://www.oculus.com/rift/ with resolution of the screen $1280 \times 800$ pxl, 110 diagonal field of view, and refresh rate of $60 \mathrm{~Hz}$. Conditions (body, object, visual only) order was randomized for each subject.

\subsection{Data analysis}

In order to study the impact of visuo-tactile conflict on the content of visual consciousness we calculated mean reaction times (RTs) to report breaks in continuous flash suppression (i.e. the time required for the target to become visible (Jiang, et al., 2007; Mudrik, et al., 2011; Stein, et al., 2012). Trials with incorrect target discrimination (corresponding to $3.6 \%$ on average across experiments), and reaction times longer than 2.5 standard deviations from the individual's mean RT, or shorter than $750 \mathrm{~ms}$ (the minimal exposure time for a perceivable tactile stimulation) were excluded from further analyses (corresponding to $8.6 \%$ of correct trials on average across 
experiments). In order to reduce deviations from normality, we performed an inverse transformation of reaction times (Whelan, 2008). We performed mixed ANOVAs with experiment as between-subject factor (body CFS/ control CFS/ object CFS) and synchrony (synchronous/asynchronous visuo-tactile stroking) as within-subject factor both for RTs and accuracy, followed by t-test planned contrasts. All statistical tests were two-sided unless otherwise stated. Data from experiment 4 was analysed using a 2 (body/diffeomorphic object) x 2 (synchronous/asynchronous visuo-tactile stroking) repeated measures ANOVA. The effects of multisensory vs. unisensory stimulation were analysed using a one-way repeated measures ANOVA including RTs in all experimental conditions (synchronous body/asynchronous body/synchronous object/asynchronous object/visual only) both for RTs and accuracy, followed by $\mathbf{t}$ tests for planned contrasts. For a better estimation of effect sizes we re-inverted reaction times and reported effect sizes in milliseconds rather than the inversed unit. Statistical analyses were performed using R ( R Foundation for Statistical Computing, Vienna, Austria).

\section{Results}

\section{Experiments 1-3}

\section{RTs}

After removing incorrect trials and outlier reaction times we conducted a mixed ANOVA to compare reaction times across conditions and experiments. We found a main effect of experiment $\left(\mathrm{F}_{(2,43)}=72.08, \mathrm{p}<0.001\right.$, partial eta square $\left.\mathrm{y}^{2}=0.77\right)$, revealing that average reaction times (reflecting the time before conscious access) were overall longer in the object CFS condition (5951ms $\pm 519 \mathrm{~ms} 95 \%$ confidence interval) compared to the body CFS condition $(1930 \mathrm{~ms} \pm 313 \mathrm{~ms})$ and control condition $(1070 \mathrm{~ms} \pm 74 \mathrm{~ms})$. We also found a main effect of condition $\left(\mathrm{F}_{(1,43)}=19.58, \mathrm{p}<0.001, \mathrm{y}^{2}=0.31\right)$, revealing that reaction times were shorter (i.e. suppression was broken faster) in the asynchronous visuo-tactile condition $(2958 \mathrm{~ms} \pm 689 \mathrm{~ms})$ compared to the synchronous visuo-tactile condition $(3105 \mathrm{~ms} \pm 706 \mathrm{~ms})$. Crucially, an interaction between synchrony and experiment $\left(\mathrm{F}_{(2,43)}=6.514, \mathrm{p}=0.003, \mathrm{y}^{2}=0.23\right)$ suggested that the presence of a visuo-tactile conflict had a different incidence depending on the experiment type. 
To test what was driving this interaction, we computed a synchrony effect index (synchronous RT-asynchronous RT) for each subject in each experiment. We then explored this effect by running planned comparisons for the synchrony effect index RTs in each experiment to directly compare the body CFS with the object CFS experiment, and the body CFS with the control CFS experiment. We found a significant difference depending on the synchrony of visuo-tactile stroking both between the body CFS and the object CFS $\left(t_{(29)}=-3.30, p=0.003\right)$ and between the body CFS and the control CFS $\left(t_{(28)}=-2.22, p=\right.$ 0.03); moreover the body experiment showed the largest effect, with largest $\mathrm{RT}$ differences between the synchronous and asynchronous conditions.

We then examined if the RT synchrony effects were significant within each experiment. As predicted, in the body experiment (body CFS) RTs in the asynchronous condition (1828ms \pm $417 \mathrm{~ms}$ ) were significantly shorter than in the synchronous condition $(2034 \mathrm{~ms}, \pm 478 \mathrm{~ms} 95 \%$ confidence interval; $\left.\mathrm{t}_{(14)}=3.8, \mathrm{p}=.002\right)$. Accordingly, $87 \%$ of the participants $(13 / 15)$ showed shorter times to break suppression when the masked visual stimuli and tactile stimulation were temporally asynchronous than synchronous, with a mean difference of $206 \mathrm{~ms}$. The difference between the synchronous and asynchronous conditions was not significant in the control experiment (control CFS: synchronous: $1081 \mathrm{~ms} \pm 110 \mathrm{~ms}$; asynchronous: $1059 \mathrm{~ms} \pm 103 \mathrm{~ms}$; $\left.t_{(14)}=1.9, p=0.08\right)$ nor in the object experiment (object CFS: synchronous: $6057 \mathrm{~ms} \pm 733 \mathrm{~ms}$; asynchronous: $\left.\mathbf{5 8 4 5 m s} \pm \mathbf{7 5 4 m s} ; \mathbf{t}_{(\mathbf{1 5})}=\mathbf{1 . 2}, \mathbf{p}=\mathbf{0 . 2 4}\right)$. For mean RTs in synchronous and asynchronous conditions in all three experiments, see Figure 2. Further analyses using RT normalization procedures yielded identical results (see Supplementary materials for details). 


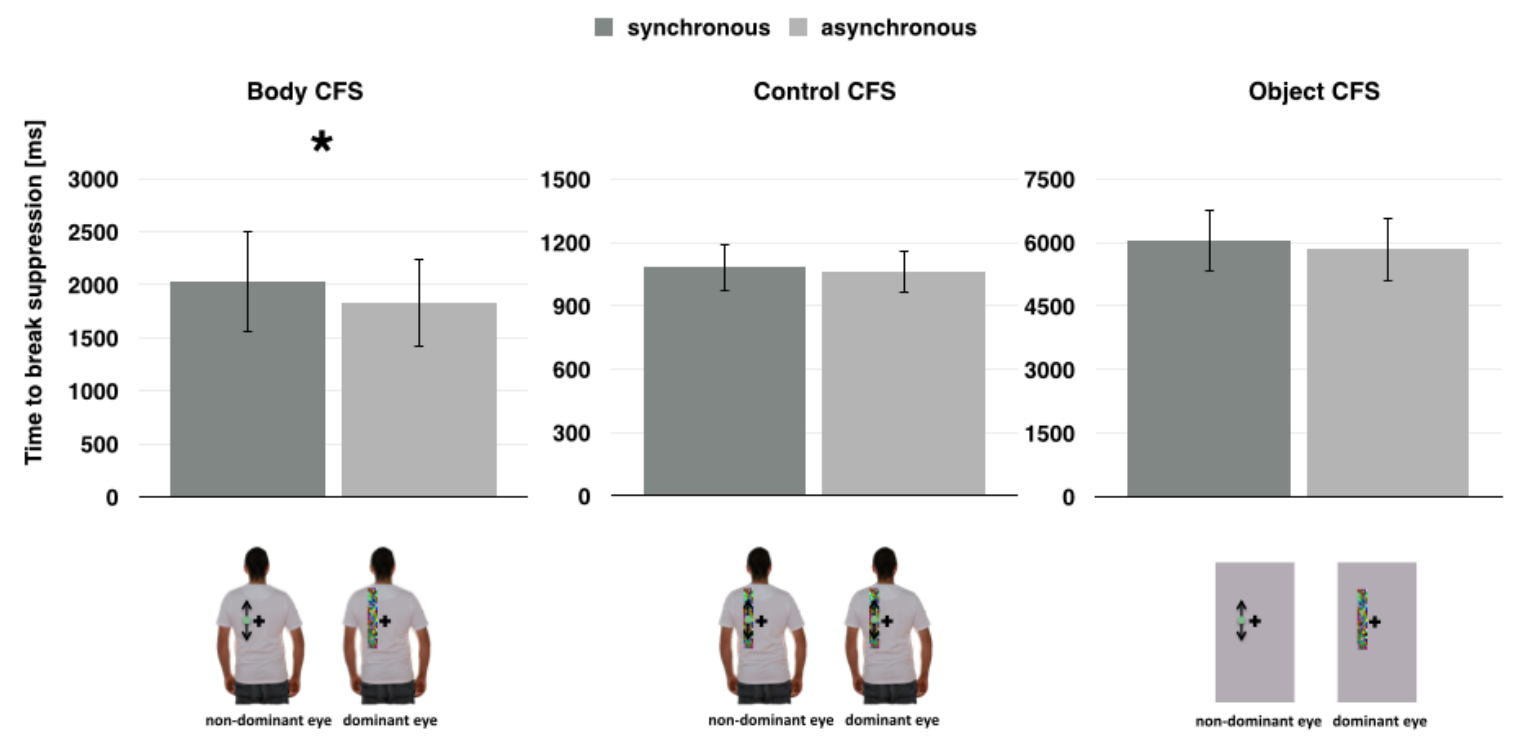

Figure 2 Mean time to break suppression (RTs) in the three experiments (body CFS, control CFS, object CFS). Comparison of RTs (in ms) between synchronous and asynchronous conditions revealed that in the body CFS experiment RTs is longer under the synchronous visuotactile stimulation comparing to the asynchronous one. Significant differences were absent in the control and object CFS $(* \mathrm{p}<.05 ; 95 \%$ Confidence Interval bars).

\section{Accuracy}

Overall accuracy in the three experiments was above $90 \%$ (body CFS $=94.38 \% \pm 2.63$, control $\mathrm{CFS}=98.46 \% \pm 0.59$, object $\mathrm{CFS}=96.08 \% \pm 2.3$ ). The mixed ANOVA revealed no significant main effect of experiment $\left(\mathrm{F}_{(2,43)}=1.88, \mathrm{p}=0.16, \mathrm{y}^{2}=.08\right)$; no significant main effect of synchrony $\left(\mathrm{F}_{(1,43)}=0.038, \mathrm{p}=0.84, \mathrm{n}^{2}<.01\right)$ nor interaction $\left(\mathrm{F}_{(2,43)}=0.21, \mathrm{p}=0.80, \mathrm{n}^{2}=.01\right)$. A paired-samples $\mathrm{t}-$ test comparison of accuracy between synchronous and asynchronous conditions did not reveal any significant differences in any of the three experiments (all p>.27). 


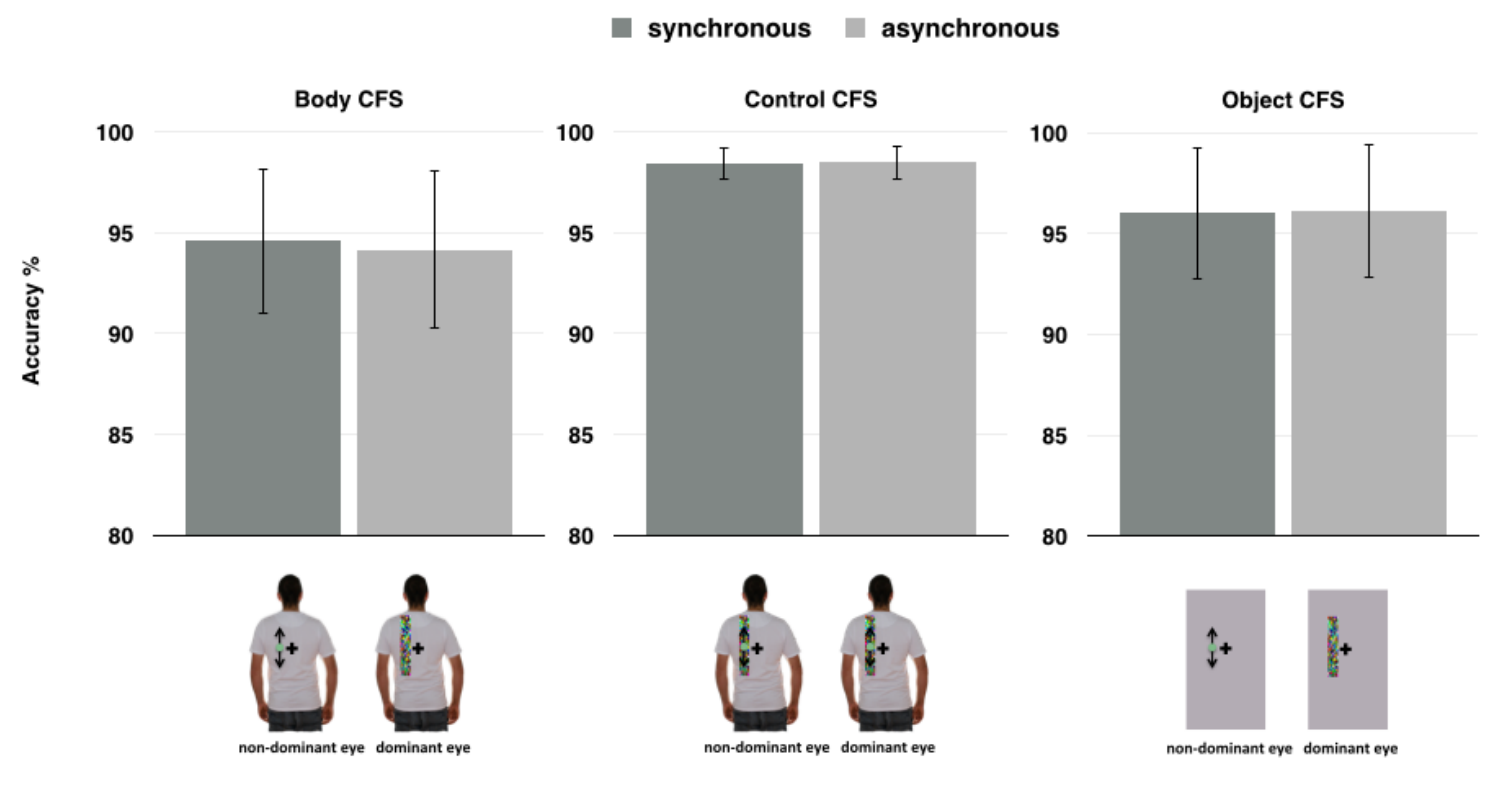

Figure 3 Accuracy in the three experiments (body CFS, control CFS, object CFS). Comparison of accuracy (in percentage) between synchronous and asynchronous conditions did not reveal any significant difference in any of the three experiments (all $p>.40 ; 95 \%$ Confidence Interval bars).

\section{Experiment 4}

As reaction times were much longer in the object condition compared to the body condition, we estimated that we insufficiently controlled for low-level perceptual differences between the object and body stimuli. Thus, we conducted an additional experiment using a within-subject design and a new object stimulus created by a diffeomorphic transformation of the body image (Stojanoski \& Cusack, 2014). We also added a unimodal visual condition (i.e. without tactile stimulation) to check whether visuotactile stimulation changes sensory processing and affects access of visual stimuli to consciousness as compared to unisensory stimulation.

\section{RTs}

After removing incorrect trials (6.6\% of trials) and outlier reaction times $(1.75 \%$ of trials) we conducted a repeated-measures ANOVA to compare reaction times across conditions. The analysis showed no effect of synchrony $\left(F_{(1,17)}=0.8 p=.38\right.$, n.s.) nor any main effect of 
visual context $\left(F_{(1,17)}=1.11, p=0.3\right.$, n.s. $)$ revealing that the $R$ Ts for the new diffeomorphic object image were not longer than in the body CFS condition. Importantly, the interaction between synchrony and visual context was significant $\left(F_{(1,17)}=5.49, p=0.03, \eta^{2}=0.24\right)$ showing that synchrony had a different effect depending on the visual context (see: Figure 4). We further explored this effect by running planned comparisons for each condition to directly compare the RTs in the body and diffeomorphic object conditions as a function of visuo-tactile synchrony. As predicted, in the body condition RTs in asynchronous condition $(4719 \mathrm{~ms} \pm 1178 \mathrm{~ms})$ were significantly shorter than in the synchronous condition $(4936 \mathrm{~ms}, \pm$ $1217 \mathrm{~ms} 95 \%$ confidence interval; $t_{(17)}=1.89, p=.037$, one tailed). In the object condition a

trend for the opposite result emerged $\left(t_{(17)}=2.01, p=.06\right.$ two tailed) with shorter $R$ Ts in the synchronous condition $(4793 \mathrm{~ms}, \pm 1061 \mathrm{~ms})$ compared to the asynchronous condition $(5105 \mathrm{~ms} \pm 906 \mathrm{~ms})$. Further analyses using RT normalization procedures yielded similar results (see Supplementary materials for details). For mean RTs and accuracy in synchronous and asynchronous conditions in all three experiments, see Figure 4. The oneway repeated measures ANOVA including RTs in all experimental conditions (synchronous body/asynchronous body/synchronous object/asynchronous object/visual only) revealed no differences in overall RTs between the multisensory and visual only conditions $\left(\mathrm{F}_{(4,68)}=\mathbf{0 . 9 2}, \mathrm{p}=0.4\right.$, n.s. $)$.

\section{Accuracy}

Overall accuracy in the three conditions was above $90 \%(\operatorname{body}=94.15 \% \pm 2.63$, diffeomorphic object $=96.19 \% \pm 0.59$, visual only $=98.33 \% \pm 2.48$ ). The repeated measure ANOVA revealed no significant main effect of synchrony $\left(F_{(1,17)}=0.08, p=0.76, \eta^{2}=.005\right)$, no significant main effect of visual context $\left(F_{(1,17)}=0.63, p=0.43, \eta^{2}=.003\right)$ nor interaction $\left(F_{(1,17)}=3.5, p=0.08, \eta^{2}=.17\right)$. 


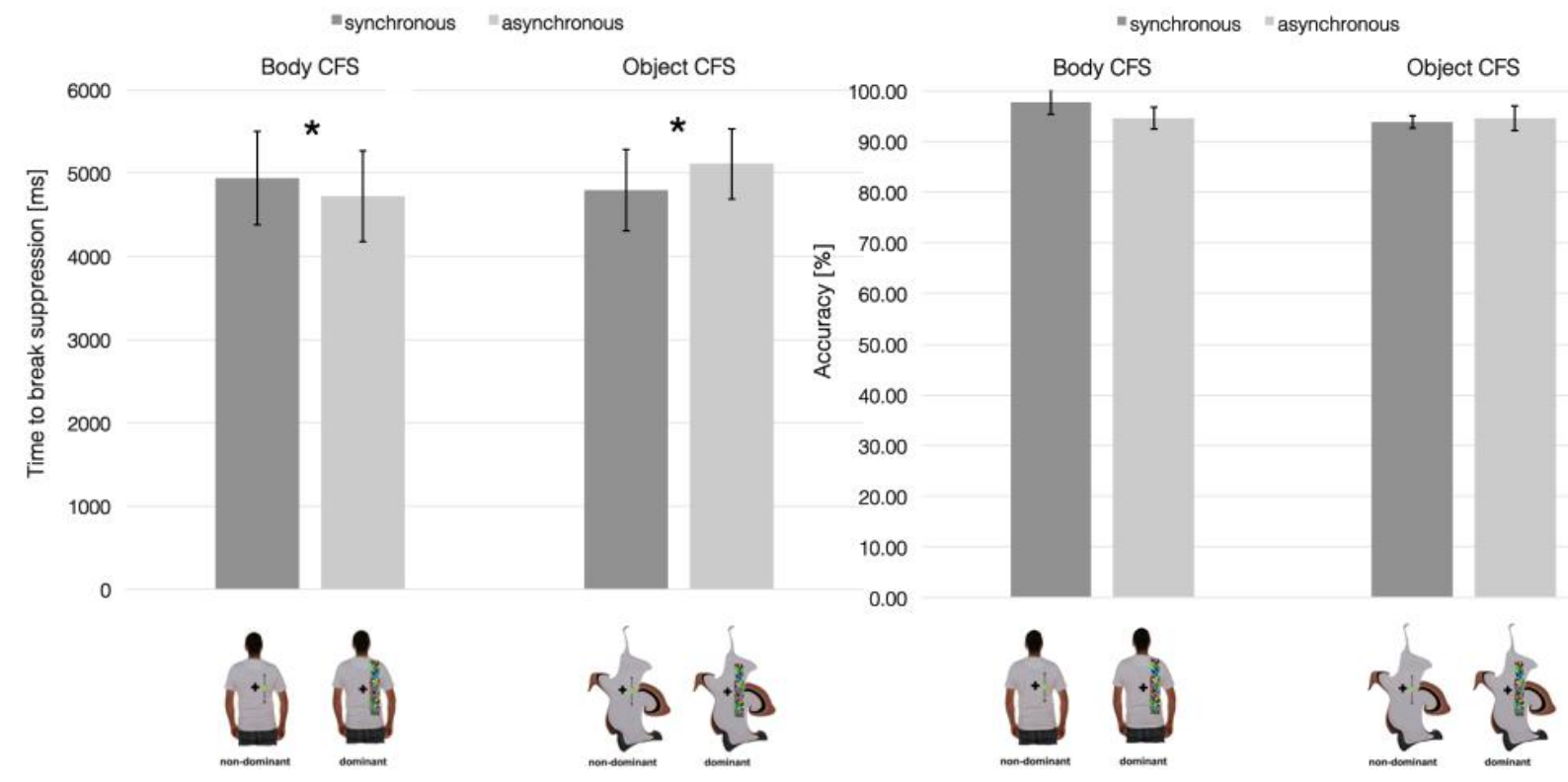

Figure 4. Reaction times and accuracy in experiment 4. Left, reaction times for synchronous and asynchronous conditions for the body and diffeomorphic visual contexts. Right, accuracy for synchronous and asynchronous conditions for the body and diffeomorphic visual contexts $(* \mathbf{p}<.05 ; 95 \%$ Confidence Interval bars).

\section{Discussion}

The present study investigated the effects of body-related visuo-tactile conflicts on visual consciousness. The results revealed three main findings: (i) body-related visuo-tactile conflicts modulated access to visual consciousness; (ii) this effect depended on the synchrony and the visual context of the stimulation; (iii) the modulation of visual consciousness occurred even when the tactile stimulation was passive and task irrelevant.

Our results indicate that the modulation of visual consciousness by passive visuo-tactile stimulation depends on the synchrony and the visual context. Longer times for conscious access were found when stimulation was synchronous versus asynchronous and presented in the context of a body, while no such suppression was found when the same tactile 
stimulation was depicted on an object. This suggests that this modulation is strokingdependent and body-specific. Interestingly, the presence of a bodily context is important for the induction of changes in bodily self-consciousness in both the rubber-hand illusion (Botvinick \& Cohen, 1998; Costantini \& Haggard, 2007; Ehrsson, Holmes, \& Passingham, 2005; Tsakiris \& Haggard, 2005) and in the full-body illusion (Aspell, et al., 2009; Ehrsson, 2007; Lenggenhager, et al., 2007; Petkova \& Ehrsson, 2008), since these modulations of BSC are typically found only when the synchronous visuo-tactile stimuli are presented on an image of a body or limb but not a control object. Thus, our present findings extend this effect to visual consciousness, by showing that body-related conflicting visuo-tactile stimulation modulates conscious access to a visual stimulus according to the context in which it is presented. Previous work has already identified some influences of visual bodily information on tactile processing (Cardini, et al., 2011; Salomon, et al., 2012; Serino, Pizzoferrato, \& Ladavas, 2008). The presentation of a body form is known to enhance the spatial acuity of touch (Cardini, et al., 2011; Cardini, Haggard, \& Ladavas, 2013; Kennett, et al., 2001) and to reduce the perceived intensity of acute pain (Longo, Betti, Aglioti, \& Haggard, 2009; Mancini, Longo, Kammers, \& Haggard, 2011; Romano, Pfeiffer, Maravita, \& Blanke, 2014). Here, the visual context in which the visual target was presented (body vs. object) modulated the time to break suppression as a function of the visuo-tactile synchrony. Synchronous stroking (inducing a body-related multisensory conflict - I feel touch on my back but see synchronous touch on the avatar's back) compared to asynchronous stroking (without a body-related multisensory conflict) caused longer times to break suppression in the context of a body image. While synchronous stroking on a non-body image (hence without a body-related multisensory conflict) did not cause an elongation of RTs.

These results show that multisensory perception, that has been proposed to be a key factor for the sense of the bodily self or BSC (Bermúdez, Marcel, \& Eilan, 1998; Blanke, 2012; Ehrsson, 2012; Gallagher \& Meltzoff, 1996), also affects visual consciousness. Previous studies have shown that BSC arises though the continuous integration of multiple sensory stimuli leading to robust own body representations for BSC (Aspell, et al., 2009; Ehrsson, et al., 2005; Lopez, Halje, \& Blanke, 2008; Pfeiffer, et al., 2013; Suzuki, Garfinkel, Critchley, \& Seth, 2013; Tsakiris \& Haggard, 2005). Accordingly, conflicting multisensory signals in a visual bodily context as used in the present study are not properly integrated 
within such body representations and are held to induce bodily illusions for hand, face, or full-body (Apps \& Tsakiris, 2014; Costantini \& Haggard, 2007; Seth, Suzuki, \& Critchley, 2011; Tsakiris \& Haggard, 2005). Here we show that, the same multisensory conflict producing bodily illusions, in particular those employed in full-body illusions (Ionta, et al., 2011; Salomon, Lim, Pfeiffer, et al., 2013) causes visual stimuli to take longer to break into awareness, compared to non-conflicting signals. One interpretation is that there is preferential cortical processing for non-conflicting multisensory signals, even when these are not consciously perceived, which thus facilitates their entrance to awareness. This is in line with several studies showing dominance of such non-conflicting multisensory stimuli in visual awareness (Aller, Giani, Conrad, Watanabe, \& Noppeney, 2015; Alsius \& Munhall, 2013; Faivre, et al., 2015; Lunghi, et al., 2010; Lunghi, et al., 2014; Maruya, Yang, \& Blake, 2007; Salomon, et al., 2015; Salomon, Lim, Herbelin, et al., 2013).

Contrary to early accounts of visual consciousness and visual information processing suggesting that the visual system is modular and encapsulated (Fodor, 1983; Zeki \& Bartels, 1998) and that multisensory integration requires consciousness (Baars, 2002) our results support recent findings (Lunghi \& Alais, 2013; Lunghi, et al., 2010; Lunghi, et al., 2014; van Ee, van Boxtel, Parker, \& Alais, 2009) showing that tactile information is integrated with visual information and affects conscious access during binocular rivalry (for related work on auditory effects on visual consciousness and olfactory effects on visual consciousness see (Conrad, et al., 2010; Faivre, et al., 2014; Zhou, et al., 2010). As the visual information presented to the observer was identical in the synchronous and asynchronous conditions the difference in suppression cannot be due to any disparity in the visual stimuli per se, but must be related to visuo-tactile coupling. Previous investigations of visual biases depending on touch have shown that tactile information may play a role for disambiguating visual information (Blake, Sobel, \& James, 2004; Butz, Thomaschke, Linhardt, \& Herbort, 2010). For example, Lunghi and colleagues have shown, using binocular rivalry, that active haptic exploration of a tactile stimulus congruent with a current visual stimulus prolonged the maintenance of the visual percept, while incongruent tactile information increased the probability of perceptual switches. Moreover, this effect was sensitive to the specific matching of spatial frequencies between the haptic and visual images (Lunghi, et al., 2010). However, our results differ considerably from those of Lunghi and colleagues as we showed that even passive and task-irrelevant tactile stimulation on the back modulates the 
conscious access to continuously suppressed visual stimuli. Thus, multisensory information is shown to be integrated in the absence of perceptual awareness, often facilitating the experience of congruent crossmodal stimuli.

While the aim of this study was to investigate if multisensory conflicts shown to modulate BSC also affect visual awareness, we also examined if the multisensory conditions affected RTs in relation to unimodal (visual only) stimulation (Exp.4). Previous studies have shown enhanced awareness for congruent multisensory conditions compared to unimodal visual only stimulation (Lunghi \& Alais, 2013). Comparing RTs in the multisensory conditions vs. unimodal visual condition in the present study revealed no significant differences, suggesting that the multisensory stimulation did not enhance or suppress access to awareness per se (Stein, 2012; Wallace, et al., 2004) but rather granted a faster conscious access to visuo-tactile signals when they were congruent, and presented in a bodily context. This might be due to the fact that in our design, the tactile stimulation was passively delivered by the robot and task irrelevant. This may reduce facilitation or inhibition related to multisensory processing of the visual and tactile stimuli compared to paradigms using active haptic exploration (e.g. Lunghi \& Alais, 2013, 2015). Although congruency effects for passive visuo-tactile stimulation in binocular rivalry were recently reported (Lunghi \& Morrone, 2013), the methods of this latter study were very different from the present study and included both passive and active conditions which may have caused residual attention to be allotted to the tactile stimuli even in the passive conditions. Furthermore, in the current study which was based on the FBI paradigm (Ionta, et al., 2011; Salomon, Lim, Pfeiffer, et al., 2013) there was no spatial correspondence between visual stimuli (viewed in front of the subjects) and the tactile stimulation (felt on the back) which may have reduced multisensory facilitation and inhibition. Future studies should investigate this issue directly.

In the control experiment (Exp.2) in which the stimuli were displayed to both eyes (no interocular suppression), no difference between the two visuo-tactile stimulation conditions was found. This suggests that the results in the body CFS experiment were not due to a response or detection bias (Jiang, et al., 2007; Mudrik, et al., 2011; Salomon, Lim, Herbelin, et al., 2013). Thus, the present modulation of visual consciousness by body-related visuo-tactile conflicts in 
the presence of a body form shows that the interactions between visual and tactile signals are important not only for bodily self-consciousness (Ehrsson, 2007; Lenggenhager, et al., 2007), but also for the formation of visual consciousness (Blake, et al., 2004; Lunghi, et al., 2010).

The brain mechanisms by which body-related visuo-tactile stimulation modulates visual consciousness are unclear. Even though no imaging data has been collected in the present study, some speculation regarding the involved cortical mechanisms seems merited. It has been suggested that the integration of multisensory signals underlying bodily self-consciousness, such as body-related visuo-tactile stimuli shown here, are driven by bimodal neurons (i.e. responsive to both tactile and visual stimulation) in premotor, parietal, and posterior temporal regions (Blanke, 2012; Ehrsson, et al., 2004; Makin, Holmes, \& Zohary, 2007). Such bimodal visuotactile neurons have been reported in non-human primates and have been linked to representation of space near the body (Bremmer, Klam, Duhamel, Ben Hamed, \& Graf, 2002; Duhamel, Colby, \& Goldberg, 1998; Graziano, Hu, \& Gross, 1997). Neuroimaging in human subjects have shown several brain regions in frontal, parietal, and temporo-occipital cortices, which respond to both tactile and visual stimulation (Calvert, 2001; Cardini, et al., 2011; Gentile, Petkova, \& Ehrsson, 2011; Makin, et al., 2007). Two candidate regions for such integration of tactile and visual signals are the extrastriate body area (EBA) and parietal regions such as the temporo-parietal junction. The EBA responds to visual images of the body and body parts (Downing, Jiang, Shuman, \& Kanwisher, 2001)and is, along with the TPJ, part of the network important for bodily self-consciousness (Arzy, Thut, Mohr, Michel, \& Blanke, 2006; Ionta, et al., 2011; Ionta, Martuzzi, Salomon, \& Blanke, 2014). This region is also sensitive to the synchrony of visuotactile stimulation (Ionta, et al., 2011) and sensorimotor conflicts (Astafiev, Stanley, Shulman, \& Corbetta, 2004)when projected on a body. Considering that CFS has been shown to impact visual representations at early stages (i.e. primary visual cortex (Yuval-Greenberg \& Heeger, 2013) one could speculate that the bimodal regions described above may play a modulating role on visual consciousness through feedback connections with visual cortex selectively strengthening neural representations of target stimuli and fastening its access to consciousness. However, further studies are needed to determine the neural mechanisms of tactile modulation of visual consciousness. 


\section{Concluding remarks}

Visual consciousness and bodily self-consciousness have been studied in relative isolation with few attempts to test the effect of bodily signals on visual consciousness. Previous work has found that proprioceptive information modulates suppression time in a continuous flash suppression task (Salomon, Lim, Herbelin, et al., 2013). Others have shown that in binocular rivalry, tactile stimuli congruent with a current visual percept (Lunghi, et al., 2010) as well as voluntarily evoked changes in visual stimuli (Maruya, et al., 2007) prolonged maintaining the percept, while incongruent increased the probability of switching it. Taken together, these studies suggest that bodily and unconsciously processed visual signals can be integrated into multimodal representations, and that signal's congruency facilitates conscious access. Here we extend this by showing that the visual context affects whether a given visuo-tactile stimulation is congruent or conflicting. A body-related visuo-tactile conflict, defined by tactile signals synchronous to an unseen visual stimulus displayed on a visible body image, elicits longer suppression times than the same stimuli presented with no visuo-tactile synchrony. Our results link visuo-tactile stimulation, when presented in the context of a body, to modulation of visual perceptual consciousness, indicating that similar factors affect both visual and bodily selfconsciousness and opening the possibility that similar mechanisms underlie both of these effects.

\section{Funding Information}

O.B. is supported by the Bertarelli Foundation, the Swiss National Science Foundation, and the European Science Foundation. R.S. was supported by the National Center of Competence in Research (NCCR) "SYNAPSY - The Synaptic Bases of Mental Diseases" financed by the Swiss

National Science Foundation ( ${ }^{\circ}$ 51AU40_125759). M.Ł. was supported by the National Science Centre, Poland (Sonata Bis Program, grant 2012/07/E/HS6/01037, PI: Michał Wierzchoń).

\section{References}

Aller, M., Giani, A., Conrad, V., Watanabe, M., \& Noppeney, U. (2015). A spatially collocated sound thrusts a flash into awareness. Frontiers in integrative neuroscience, 9.

Alsius, A., \& Munhall, K. G. (2013). Detection of audiovisual speech correspondences without visual awareness. Psychological Science, 24, 423-431. 
Apps, M. A., \& Tsakiris, M. (2014). The free-energy self: a predictive coding account of selfrecognition. Neuroscience \& Biobehavioral Reviews, 41, 85-97.

Arzi, A., Shedlesky, L., Ben-Shaul, M., Nasser, K., Oksenberg, A., Hairston, I. S., \& Sobel, N. (2012). Humans can learn new information during sleep. Nature Neuroscience, 15, 14601465.

Arzy, S., Thut, G., Mohr, C., Michel, C. M., \& Blanke, O. (2006). Neural basis of embodiment: distinct contributions of temporoparietal junction and extrastriate body area. J Neurosci, 26, 8074-8081.

Aspell, J. E., Heydrich, L., Marillier, G., Lavanchy, T., Herbelin, B., \& Blanke, O. (2013). Turning body and self inside out: visualized heartbeats alter bodily self-consciousness and tactile perception. Psychol Sci, 24, 2445-2453.

Aspell, J. E., Lenggenhager, B., \& Blanke, O. (2009). Keeping in touch with one's self: multisensory mechanisms of self-consciousness. PLoS ONE, 4, e6488.

Astafiev, S. V., Stanley, C. M., Shulman, G. L., \& Corbetta, M. (2004). Extrastriate body area in human occipital cortex responds to the performance of motor actions. Nat Neurosci, 7, 542-548.

Baars, B. J. (2002). The conscious access hypothesis: origins and recent evidence. Trends Cogn Sci, 6, 47-52.

Bayne, T. (2002). The unity of consciousness. In L. Nadel (Ed.), Encyclopedia of Cognitive Science (pp. 786-793): Nature Publishing Group.

Bermúdez, J. L., Marcel, A. J., \& Eilan, N. (1998). The body and the self: The MIT Press.

Blake, R., Sobel, K. V., \& James, T. W. (2004). Neural synergy between kinetic vision and touch. Psychol Sci, 15, 397-402.

Blanke, O. (2012). Multisensory brain mechanisms of bodily self-consciousness. Nat Rev Neurosci, 13, 556-571.

Blanke, O., Pozeg, P., Hara, M., Heydrich, L., Serino, A., Yamamoto, A., Higuchi, T., Salomon, R., Seeck, M., Landis, T., Arzy, S., Herbelin, B., Bleuler, H., \& Rognini, G. (2014). Neurological and robot-controlled induction of an apparition. Curr Biol, 24, 2681-2686.

Botvinick, M., \& Cohen, J. (1998). Rubber hands 'feel' touch that eyes see. Nature, 391, 756.

Bremmer, F., Klam, F., Duhamel, J. R., Ben Hamed, S., \& Graf, W. (2002). Visual-vestibular interactive responses in the macaque ventral intraparietal area (VIP). Eur J Neurosci, 16, 1569-1586.

Butz, M. V., Thomaschke, R., Linhardt, M. J., \& Herbort, O. (2010). Remapping motion across modalities: tactile rotations influence visual motion judgments. Exp Brain Res, 207, 1-11.

Calvert, G. A. (2001). Crossmodal processing in the human brain: insights from functional neuroimaging studies. Cerebral Cortex, 11, 1110-1123.

Cardini, F., Costantini, M., Galati, G., Romani, G. L., Làdavas, E., \& Serino, A. (2011). Viewing one's own face being touched modulates tactile perception: An fmri study. Journal of cognitive neuroscience, 23, 503-513.

Cardini, F., Haggard, P., \& Ladavas, E. (2013). Seeing and feeling for self and other: proprioceptive spatial location determines multisensory enhancement of touch. Cognition, 127, 84-92.

Conrad, V., Bartels, A., Kleiner, M., \& Noppeney, U. (2010). Audiovisual interactions in binocular rivalry. Journal of Vision, 10, 27.

Costantini, M., \& Haggard, P. (2007). The rubber hand illusion: Sensitivity and reference frame for body ownership. Consciousness and Cognition, 16, 229-240. 
Dehaene, S., \& Changeux, J. P. (2011). Experimental and Theoretical Approaches to Conscious Processing. Neuron, 70, 200-227.

Dehaene, S., Changeux, J. P., Naccache, L., Sackur, J., \& Sergent, C. (2006). Conscious, preconscious, and subliminal processing: a testable taxonomy. Trends Cogn Sci, 10, 204211.

Deroy, O., Chen, Y. C., \& Spence, C. (2014). Multisensory constraints on awareness. Philos Trans R Soc Lond B Biol Sci, 369, 20130207.

Downing, P. E., Jiang, Y., Shuman, M., \& Kanwisher, N. (2001). A cortical area selective for visual processing of the human body. Science, 293, 2470-2473.

Driver, J., \& Noesselt, T. (2008). Multisensory interplay reveals crossmodal influences on 'sensory-specific' brain regions, neural responses, and judgments. Neuron, 57, 11-23.

Dubois, J., \& Faivre, N. (2014). Invisible, but how? The depth of unconscious processing as inferred from different suppression techniques. Frontiers in Psychology, 5.

Duenas, J., Chapuis, D., Pfeiffer, C., Martuzzi, R., Ionta, S., Blanke, O., \& Gassert, R. (2011). Neuroscience robotics to investigate multisensory integration and bodily awareness. Conf Proc IEEE Eng Med Biol Soc, 2011, 8348-8352.

Duhamel, J. R., Colby, C. L., \& Goldberg, M. E. (1998). Ventral intraparietal area of the macaque: congruent visual and somatic response properties. J Neurophysiol, 79, 126-136.

Ehrsson, H. H. (2007). The experimental induction of out-of-body experiences. Science, 317, 1048.

Ehrsson, H. H. (2012). The Concept of Body Ownership and Its Relation to Multisensory Integration.

Ehrsson, H. H., Holmes, N. P., \& Passingham, R. E. (2005). Touching a rubber hand: feeling of body ownership is associated with activity in multisensory brain areas. J Neurosci, 25, 10564-10573.

Ehrsson, H. H., Spence, C., \& Passingham, R. E. (2004). That's my hand! Activity in premotor cortex reflects feeling of ownership of a limb. Science, 305, 875-877.

Evans, N., \& Blanke, O. (2012). Shared electrophysiology mechanisms of body ownership and motor imagery. Neuroimage.

Faivre, N., Mudrik, L., Schwartz, N., \& Koch, C. (2014). Multisensory Integration in Complete Unawareness Evidence From Audiovisual Congruency Priming. Psychological Science, 25, 2006-2016.

Faivre, N., Salomon, R., \& Blanke, O. (2015). Visual consciousness and bodily selfconsciousness. Current opinion in neurology, 28, 23-28.

Fodor, J. A. (1983). The modularity of mind: An essay on faculty psychology. Cambridge: MIT Press.

Gallagher, S., \& Meltzoff, A. (1996). The earliest sense of self and others: Merleau-Ponty and recent developmental studies. Philosophical psychology, 9, 211-233.

Gayet, S., Van der Stigchel, S., \& Paffen, C. L. E. (2014). Breaking continuous flash suppression: competing for consciousness on the pre-semantic battlefield. Frontiers in Psychology, 5.

Gentile, G., Petkova, V. I., \& Ehrsson, H. H. (2011). Integration of visual and tactile signals from the hand in the human brain: an FMRI study. J Neurophysiol, 105, 910-922.

Graziano, M. S., Hu, X. T., \& Gross, C. G. (1997). Visuospatial properties of ventral premotor cortex. J Neurophysiol, 77, 2268-2292. 
Hesselmann, G., \& Malach, R. (2011). The Link between fMRI-BOLD Activation and Perceptual Awareness Is "Stream-Invariant" in the Human Visual System. Cerebral Cortex, 21, 2829-2837.

Ionta, S., Heydrich, L., Lenggenhager, B., Mouthon, M., Fornari, E., Chapuis, D., Gassert, R., \& Blanke, O. (2011). Multisensory Mechanisms in Temporo-Parietal Cortex Support SelfLocation and First-Person Perspective. Neuron, 70, 363-374.

Ionta, S., Martuzzi, R., Salomon, R., \& Blanke, O. (2014). The brain network reflecting bodily self-consciousness: a functional connectivity study. Soc Cogn Affect Neurosci.

Jiang, Y., Costello, P., \& He, S. (2007). Processing of invisible stimuli: Advantage of upright faces and recognizable words in overcoming interocular suppression. Psychological Science, 18, 349-355.

Kennett, S., Taylor-Clarke, M., \& Haggard, P. (2001). Noninformative vision improves the spatial resolution of touch in humans. Curr Biol, 11, 1188-1191.

Kim, C. Y., \& Blake, R. (2005). Psychophysical magic: rendering the visible 'invisible'. Trends Cogn Sci, 9, 381-388.

Kouider, S., \& Dehaene, S. (2007). Levels of processing during non-conscious perception: a critical review of visual masking. Philosophical Transactions of the Royal Society BBiological Sciences, 362, 857-875.

Lenggenhager, B., Tadi, T., Metzinger, T., \& Blanke, O. (2007). Video ergo sum: manipulating bodily self-consciousness. Science, 317, 1096-1099.

Longo, M. R., Betti, V., Aglioti, S. M., \& Haggard, P. (2009). Visually induced analgesia: seeing the body reduces pain. J Neurosci, 29, 12125-12130.

Lopez, C., Halje, P., \& Blanke, O. (2008). Body ownership and embodiment: vestibular and multisensory mechanisms. Neurophysiologie Clinique/Clinical Neurophysiology, 38, 149-161.

Lunghi, C., \& Alais, D. (2013). Touch interacts with vision during binocular rivalry with a tight orientation tuning. PLoS ONE, 8, e58754.

Lunghi, C., \& Alais, D. (2015). Congruent tactile stimulation reduces the strength of visual suppression during binocular rivalry. Sci. Rep., 5.

Lunghi, C., Binda, P., \& Morrone, M. C. (2010). Touch disambiguates rivalrous perception at early stages of visual analysis. Current Biology, 20, R143-R144.

Lunghi, C., \& Morrone, M. C. (2013). Early interaction between vision and touch during binocular rivalry. Multisensory research, 26, 291-306.

Lunghi, C., Morrone, M. C., \& Alais, D. (2014). Auditory and Tactile Signals Combine to Influence Vision during Binocular Rivalry. J Neurosci, 34, 784-792.

Makin, T. R., Holmes, N. P., \& Zohary, E. (2007). Is that near my hand? Multisensory representation of peripersonal space in human intraparietal sulcus. $J$ Neurosci, 27, 731740.

Mancini, F., Longo, M. R., Kammers, M. P., \& Haggard, P. (2011). Visual distortion of body size modulates pain perception. Psychol Sci, 22, 325-330.

Maruya, K., Yang, E., \& Blake, R. (2007). Voluntary action influences visual competition. Psychological Science, 18, 1090-1098.

Miles, W. R. (1930). Ocular dominance in human adults. Journal of General Psychology, 3, $412-$ 429.

Mudrik, L., Breska, A., Lamy, D., \& Deouell, L. Y. (2011). Integration Without Awareness: Expanding the Limits of Unconscious Processing. Psychological Science, 22, 764-770. 
Mudrik, L., Faivre, N., \& Koch, C. (2014). Information integration without awareness. Trends Cogn Sci, 18, 488-496.

Pavani, F., Spence, C., \& Driver, J. (2000). Visual capture of touch: out-of-the-body experiences with rubber gloves. Psychol Sci, 11, 353-359.

Petkova, V. I., \& Ehrsson, H. H. (2008). If I Were You: Perceptual Illusion of Body Swapping. PLOS ONE, 3.

Pfeiffer, C., Lopez, C., Schmutz, V., Duenas, J. A., Martuzzi, R., \& Blanke, O. (2013). Multisensory Origin of the Subjective First-Person Perspective: Visual, Tactile, and Vestibular Mechanisms. PLoS ONE, 8, e61751.

Rees, G. (2007). Neural correlates of the contents of visual awareness in humans. Philos Trans $R$ Soc Lond B Biol Sci, 362, 877-886.

Romano, D., Pfeiffer, C., Maravita, A., \& Blanke, O. (2014). Illusory self-identification with an avatar reduces arousal responses to painful stimuli. Behavioural Brain Research, 261, 275-281.

Salomon, R., Kaliuzhna, M., Herbelin, B., \& Blanke, O. (2015). Balancing awareness: Vestibular signals modulate visual consciousness in the absence of awareness. Consciousness and Cognition, 36, 289-297.

Salomon, R., Lim, M., Herbelin, B., Hesselmann, G., \& Blanke, O. (2013). Posing for awareness: proprioception modulates access to visual consciousness in a continuous flash suppression task. J Vis, 13, 2.

Salomon, R., Lim, M., Pfeiffer, C., Gassert, R., \& Blanke, O. (2013). Full body illusion is associated with widespread skin temperature reduction. Front Behav Neurosci, 7, 65.

Salomon, R., van Elk, M., Aspell, J., \& Blanke, O. (2012). I feel who I see: Visual body identity affects visual-tactile integration in peripersonal space. Consciousness and Cognition.

Serino, A., Pizzoferrato, F., \& Ladavas, E. (2008). Viewing a face (especially one's own face) being touched enhances tactile perception on the face. Psychological Science, 19, 434.

Seth, A. K., Suzuki, K., \& Critchley, H. D. (2011). An interoceptive predictive coding model of conscious presence. Frontiers in Psychology, 2.

Stein, B. E. (2012). The new handbook of multisensory processing: MIT Press.

Stein, T., Hebart, M. N., \& Sterzer, P. (2011). Breaking Continuous Flash Suppression: A New Measure of Unconscious Processing during Interocular Suppression? Front Hum Neurosci, 5, 167.

Stein, T., \& Sterzer, P. (2014). Unconscious processing under interocular suppression: getting the right measure. Frontiers in Psychology, 5.

Stein, T., Sterzer, P., \& Peelen, M. V. (2012). Privileged detection of conspecifics: Evidence from inversion effects during continuous flash suppression. Cognition, 125, 64-79.

Stojanoski, B., \& Cusack, R. (2014). Time to wave good-bye to phase scrambling: Creating controlled scrambled images using diffeomorphic transformations. Journal of Vision, 14.

Suzuki, K., Garfinkel, S. N., Critchley, H. D., \& Seth, A. K. (2013). Multisensory integration across exteroceptive and interoceptive domains modulates self-experience in the rubberhand illusion. Neuropsychologia, 51, 2909-2917.

Taylor-Clarke, M., Kennett, S., \& Haggard, P. (2002). Vision modulates somatosensory cortical processing. Current Biology, 12, 233-236.

Tsakiris, M., Carpenter, L., James, D., \& Fotopoulou, A. (2010). Hands only illusion: multisensory integration elicits sense of ownership for body parts but not for noncorporeal objects. Exp Brain Res, 204, 343-352. 
Tsakiris, M., \& Haggard, P. (2005). The rubber hand illusion revisited: visuotactile integration and self-attribution. J Exp Psychol Hum Percept Perform, 31, 80-91.

Tsuchiya, N., \& Koch, C. (2005). Continuous flash suppression reduces negative afterimages. Nat Neurosci, 8, 1096-1101.

van Ee, R., van Boxtel, J. J., Parker, A. L., \& Alais, D. (2009). Multisensory congruency as a mechanism for attentional control over perceptual selection. The Journal of Neuroscience, 29, 11641-11649.

van Gaal, S., \& Lamme, V. A. (2012). Unconscious high-level information processing: implication for neurobiological theories of consciousness. Neuroscientist, 18, 287-301.

Wallace, M. T., Roberson, G., Hairston, W. D., Stein, B. E., Vaughan, J. W., \& Schirillo, J. A. (2004). Unifying multisensory signals across time and space. Experimental brain research, 158, 252-258.

Weinstein, S. (1968). Intensive and extensive aspects of tactile sensitivity as a function of body part, sex and laterality. In the First Int'l symp. on the Skin Senses, 1968.

Whelan, R. (2008). Effective analysis of reaction time data. Psychological Record, 58, 475-482.

Yuval-Greenberg, S., \& Heeger, D. J. (2013). Continuous flash suppression modulates cortical activity in early visual cortex. $J$ Neurosci, 33, 9635-9643.

Zeki, S., \& Bartels, A. (1998). The autonomy of the visual systems and the modularity of conscious vision. Philos Trans R Soc Lond B Biol Sci, 353, 1911-1914.

Zhou, W., Jiang, Y., He, S., \& Chen, D. (2010). Olfaction Modulates Visual Perception in Binocular Rivalry. Current Biology, 20, 1356-1358.

\section{Figure Captions}

Figure 1. Visual stimuli in the three experiments (body CFS, control CFS, object CFS) and set up. A) body CFS: the body form and Mondrians were displayed to the dominant eye, while the moving dot was projected to the non-dominant eye (B) control CFS: the body form, Mondrians and moving dot were displayed to both eyes at the same time so that no interocular suppression occurred (C) object CFS: the object form and Mondrians were displayed to the dominant eye, while the moving dot was projected to the non-dominant eye. The black arrows indicate schematically the movement of the dot and were not present during the experiment. (D) Diffeomorphic object CFS: Object used in experiment 4 which is a diffeomorphic version of the body image thus well controlled for low level properties. (E) Schematic representation of the experimental set up. Participants were fitted with the HMD and asked to lie down supine on the stroking robot. 
Figure 2 Mean time to break suppression (RTs) in the three experiments (body CFS, control CFS, object CFS). Comparison of RTs (in ms) between synchronous and asynchronous conditions revealed that in the body CFS experiment RTs is longer under the synchronous visuotactile stimulation comparing to the synchronous one. Significant differences were absent in the control and object CFS (* $\mathrm{p}<.05 ; 95 \%$ Confidence Interval bars).

Figure 3 Accuracy in the three experiments (body CFS, control CFS, object CFS). Comparison of accuracy (in percentage) between synchronous and asynchronous conditions did not reveal any significant difference in any of the three experiments (all p>.40; 95\% Confidence Interval bars).

Figure 4 Reaction times and accuracy in experiment 4. Left, reaction times for synchronous and asynchronous conditions for the body and diffeomorphic visual contexts. Right, accuracy for synchronous and asynchronous conditions for the body and diffeomorphic visual contexts (* $\mathbf{p}<.05 ; 95 \%$ Confidence Interval bars). 